\title{
In silico analysis of a Rhizobium sp. RC1 putative haloalkanoic permease sequence motif and classification
}

\author{
Muhammed Adamu Musa and Fahrul Huyop* \\ Department of Biotechnology and Medical Engineering, Faculty of Biosciences and Medical Engineering, Universiti \\ Teknologi Malaysia, 81310 Johor, Malaysia. \\ Email: fahrul@utm.my
}

Received 18 July 2017; Received in revised form 10 August 2018; Accepted 12 August 2018

\begin{abstract}
Aims: The transport of haloalkanoic acids (haloacids) is important in the metabolism of haloacid pollutants by bacteria. In this study, a computational analysis of Rhizobium sp. RC1 haloacid permease (DehrP) amino acid sequence was conducted to identify its subfamily, sequence motifs and evolutionary position among closely related transporters.

Methodology and results: Blast search in the Pfam and Transmembrane Classification Databases was used to establish the classification and the subfamily of DehrP. Clustal omega sequence alignment approach and MEME Suite motif-based analysis tools were used to locate the transporter motifs of DehrP. Dotplots of DehrP sequence was computed using the EMBOSS Dotmatcher. MEGA7 software was used to analyze the phylogenetic position of DehrP among closely related symporters in the Transmembrane Classification Database. Comparative analysis by Pfam shows that DehrP is a member of the Major Facilitator Superfamily (\#2.A.1). PSI-Blast against the Transmembrane Classification Database shows that DehrP is significantly aligned with a subfamily of transporters called the Metabolite: $\mathrm{H}^{+}$Symporters (\#2.A.1.6). DehrP has six similar sequence motifs with the Metabolite: $\mathrm{H}^{+}$Symporter proteins including the functional motif of GXXXDRXGRR. DehrP is evolutionarily related to Burkholderia caribensis MBA4 Haloacid: $\mathrm{H}^{+}$ Symporters (Dehp2 and Deh4p).

Conclusion, significance and impact study: Based on sequence similarity, DehrP is a Major Facilitator Superfamily protein that belongs to the Metabolite: $\mathrm{H}^{+}$Symporter protein subfamily which might coordinate the transport of a haloacid coupled with a proton $\left(\mathrm{H}^{+}\right)$. Mutagenesis of DehrP sequence motifs might be useful in the engineering of Rhizobium sp. $\mathrm{RC} 1$ for efficient uptake and degradation of haloacids.
\end{abstract}

Keywords: Haloacids, haloalkanoic permease, Rhizobium sp. RC1, sequence motif, symporter

\section{INTRODUCTION}

A relatively safe and economical method of haloalkanoic acid (haloacid) pollutants removal from the environment is by microbial degradation (Su et al., 2013). However, some haloacids are difficult to degrade (Effendi et al., 2000; Berthiaume et al., 2014), partly due to the slow growth of cells (van der Ploeg and Janssen, 1995), haloacid selectivity (Berry et al., 1979; Janssen et al., 1985; Chaudhry and Chapalamadugu, 1991), and toxicity (Strotmann et al., 1990; Plewa et al., 2010). One of the important steps in the breakdown of haloacids by microorganisms is their ability to transport the pollutants into the cytoplasm (Su et al., 2013). Membrane transport can influence the Monod saturation constant $(K s)$ of microbial growth (van den Wijngaard et al., 1993) and the haloacid dehalogenation by cells (Nijenhuis et al., 2005). The idea of haloacid transporters have been suggested in bacteria such as Pseudomonas putida PP3 (Slater et al.,
1985), Xanthobacter autotrophicus GJ10 (van der Ploeg et al., 1995), Agrobacterium sp. NHG3 (Higgins et al., 2005), and Burkholderia caribensis MBA4 (Yu et al., 2007). Two distinct haloacid transporters (Deh4p and Dehp2) from $B$. caribensis MBA4 have been demonstrated to have different preferences for monochloroacetate and chloropropionate (Su and Tsang, 2013). However, most of the haloacid transporters are uncharacterized and therefore very little is known about the function of the haloacid transport proteins.

Rhizobium sp. RC1, a Gram-negative bacterium, isolated from soil using 2, 2-dichloropropionic acid as a carbon and energy source, have shown its ability to degrade different types of haloacid such as D, L-2chloropropionic acid (D, L-2-CPA), monochloroacetic acid (MCAA) and monobromoacetic acid (MBAA) (Berry et al., 1979; Cairns, 1994; Stringfellow et al., 1997). Rhizobium sp. RC1 was reported to be the only organism that synthesized three different dehalogenases (D-2-haloacid 
dehalogenase; DehD, L-2-haloacid dehalogenase; DehL and dual isomeric haloacid dehalogenase; DehE) to degrade different haloacid stereoisomers (Leigh et al., 1986; Leigh et al., 1988; Huyop, 2003). Rhizobium sp. $\mathrm{RC} 1$ haloacid permease (DehrP) has been proposed to be involved in the chloropropionic acid uptake (Jing et al., 2010). The Rhizobium sp. RC1 haloacid permease gene dehrP, with a $1239 \mathrm{~kb}$ open reading frame, is located 511 bases upstream of the $\mathrm{D}$-2-haloacid dehalogenase gene dehD, and encodes a 412 amino acids protein (DehrP) that has the Major Facilitator Superfamily (MFS) protein conserved domain (Jing et al., 2010).

In this study, computational analysis of Rhizobium sp. $\mathrm{RC} 1$ haloacid permease (DehrP) sequence was carried out, with the believe that DehrP is a specialized chloropropionate transporting protein. Comparative analysis of DehrP primary sequence in the Pfam Database and the Transmembrane Classification Database (TCDB) was done to establish its classification and the subfamily of the Major Facilitator Superfamily (MFS) it belongs, based on sequence similarity. Comparative motif analysis of DehrP sequence was done to identify functional sequence motifs using sequence alignment approach and motif-based analysis tools (MEME) (Bailey and Elkan, 1994) and (MAST) (Bailey and Gribskov, 1998). A phylogenetic tree of DehrP and Metabolite: $\mathrm{H}^{+}$Symporter family of transporters in the TCDB was constructed to reveal DehrP evolutionary position.

\section{MATERIALS AND METHODS}

\section{Sequence retrieval and primary analysis}

Rhizobium sp. RC1 haloacid permease (DehrP) (Jing et al., 2010) amino acid sequence was downloaded from the UniProtKB Database (Consortium, 2015) under the accession number Q1M2W6. NCBI-Blastp (Altschup et al., 1990), PSI-Blast (Schaffer et al., 2001) and UniProt-Blastp (Consortium, 2015) were used to analyze the amino acid sequence of DehrP. Pfam v.31.0 (Finn et al., 2016) was used to analyze the transporter classification of DehrP. Major Facilitator Superfamily (MFS) protein sequences for comparative analysis were downloaded from UniProtKB (Consortium, 2015) and the Transmembrane Classification Database (TCDB) (Saier et al., 2016).

\section{Motif analysis}

The Rhizobium sp. RC1 haloacid permease (DehrP) conserved Major Facilitator Superfamily (MFS) motif was identified by aligning with eleven MFS transporters from $E$. coli (GARP_ECOLI, TCR2_ECOLX, YJHB_ECOLI, YAAU_ECOLI, RAFB_ECOLX, LACY_ECOLI), C. freundi (LACY_CITFR), K. pneumoniae (LACY_KLEPN), Synechocystis sp. (GLCP_SYNY3), B. subtilis (ARAE_BACSU, and TCRB_BACSU) and $S$. cerevisiae (HXT2_YEAST) along the region connecting transmembrane helices 2 and 3 as described by Hirai et al. (2003) and visualized using WebLogo v.3.5 (Crooks et al., 2004). A comparative analysis of the family-specific motifs of DehrP and the twelve (12) Metabolite: $\mathrm{H}^{+}$ Symporters (MHS, class: \#2.A.1.6) family of proteins in the Transmembrane Classification Database (TCBD IDs: Dehp2; F8SVK1, Deh4p; Q7X4L6, PcaT; Q52000, KgtP; POAEX3, ShiA; P76350, ProP; P0C0L7, YhjE; P37643, ThiU; P44699, CitH; P16482, TcuC; P0A2G3, MopB; Q45082, and YdfJ; P77228) was carried out using the motif-based analysis tools MEME (Bailey and Elkan, 1994) and MAST (Bailey and Gribskov, 1998) in MEME Suite v.4.11.4.

\section{Sequence alignment}

Threshold Dot plots of the full length of DehrP sequence with six Metabolite: $\mathrm{H}^{+}$Symporters (MHS) protein sequences in the Transmembrane Classification Database (TCDB IDs: Dehp2; F8SVK1, Deh4p; Q7X4L6, ShiA; P76350, ProP; P0C0L7, YhjE; P37643, and KgtP; POAEX3) was done using Dotmatcher of the EMBOSS (Rice et al., 2000) server at a window size of 10, threshold of 23 and a default matrix. DehrP sequence was aligned with closely related haloacid transporters from Agrobacterium sp. NHG3 (DehP; Uniprot accession number: Q8KLT0) (Higgins et al., 2005) and B. caribensis MBA4 (Deh4p; Uniprot accession number: A0A0N7JV68 and Dehp2; Uniprot accession number: A0A0P0R5D5) (Yu et al., 2007; Su and Tsang, 2013) using Clustal omega (Sievers et al., 2011) and visualized using the ESPript v.3.0 (Robert and Gouet, 2014) server.

\section{Phylogenetic tree construction}

The 12 MHS sequence from the TCDB (Saier et al., 2016) was used to construct a phylogenetic tree using MEGA7 (Kumar et al., 2016) software with neighbor-joining method (Saitou and Nei, 1987). The MHS amino acid sequences were aligned by ClustalW (Larkin et al., 2007). The bootstrap values were calculated with 500 replicates (Felsenstein, 1985) and those above 50 are shown at the nodes.

\section{RESULTS AND DISCUSSION}

Primary analysis of Rhizobium sp. Rc1 haloacid permease

Membrane transporters are classified based on their structure, function, mechanisms of transport, and evolutionary position in the transporter classification databases (Finn et al., 2016; Saier et al., 2016). Comparative analysis of Rhizobium sp. RC1 haloacid permease (DehrP) primary sequence with transport proteins in the Pfam database (Finn et al., 2016) shows that DehrP is a Major Facilitator Superfamily (MFS_TC2.A.1) protein. MFS is a large group of transporters that are involved in the movement of compounds from the environmental into the cells (Mitchell, 1967; Marger and Saier, 1993; Pao et al., 1998; Reddy et al., 2012). PSI-Blast (Schaffer et al., 2001) of DehrP 
amino acid sequence against the Transporter Classification Database (TCDB) (Saier et al., 2016) turned out twelve (12) Metabolites: $\mathrm{H}^{+}$Symporters (MHS) family (\#2.A.1.6), a subfamily of the MFS transporters, that had significant sequence alignments with DehrP sequence. The 12 MHS sequences (B. caribensis MBA4 haloacid permeases; Dehp2 (class \#2.A.1.6.11) (Su and Tsang, 2013) and Deh4p (class \#2.A.1.6.8) (Yu et al., 2007), Escherichia coli Shikimate transporter; ShiA (class \#2.A.1.6.6) (Whipp et al., 1998), E. coli proline/betaine transporter; ProP (class \#2.A.1.6.4) (MacMillan et al., 1999), E. coli Osmoprotectants transporter; YhjE (class \#2.A.1.6.10) (Ly et al., 2004), E. coli a-ketoglutarate permease; KgtP (class \#2.A.1.6.2) (Seol and Shatkin, 1992), Pseudomonas putida dicarboxylate transporter; PcaT (class \#2.A.1.6.3) (Karimian and Ornston, 1981), Burkholderia cepacia Aromatic/4-methyphthalate transporter; MopB (class \#2.A.1.6.5) (Saint and Romas, 1996), Haemophilus influenzae Thiazole transporter; ThiU (class \#2.A.1.6.12) (Rodionov et al., 2002), Salmonella typhimurium Citrate-proton symporter; TcuC (class \#2.A.1.6.7) (Lewis et al., 2004), E. coli $\mathrm{K}^{+}$channel; YdfJ (class \#2.A.1.6.9) (Tang et al., 2011), and Klebsiella pneumoniae Citrate-proton symporter; $\mathrm{CitH}$ (class \#2.A.1.6.1) (Van der Rest et al., 1991). Score value of the PSI-Blast showed that Dehp2 (score: 475) and Deh4p (score: 473) were more aligned with Rhizobium sp. RC1 haloaocid permease; DehrP. Since Dehp2, Deh4p and DehrP can transport chloropropionate, therefore, DehrP might be a MHS class of \#2.A.1.6.

\section{The MFS Motifs of Rhizobium sp. RC1 haloacid permease}

Protein sequences have short motifs, mostly of a specific length, and patterns which may function as binding domains (Liò and Bishop, 2008). Comparative analyses of existing Major Facilitator Superfamily (MFS) proteins have identified specific sequence motif ([RK]XGR [RK]) for the MFS transmembrane proteins (Henderson and Maiden, 1990). The MFS motif was later extended to ten residues (GXXXDRXGRR) by Hirai et al. (2003) for the region connecting transmembrane helices (TMs) 2 and 3 . Alignment of eleven MFS sequences along the region connecting TMs 2 and 3 with DehrP sequence shows the presence of such a motif (Figure 1) at residues 83 to 92. Replacement of the MFS motif residues in $E$. coli tetracycline resistance efflux transporter (TetA, chain $B$ ) (Tamura et al., 2001) and lactose permease (LacY) (Frillingos et al., 1998) was reported to abolish transport activity.

The first-position glycine (G), fifth-position aspartic acid (D), and eighth-position glycine (G) are very important for LacY transport activity in $E$. coli (JessenMarshall et al., 1995). Further, comparative motif analysis of DehrP with the 12 MHS members using a motif-based sequence analysis programs MEME (Bailey and Elkan, 1994) and MAST (Bailey and Gribskov, 1998) shows that DehrP has common sequence motifs with the MHS proteins. Both transporters have at least six conserved motifs that are arranged in a similar fashion in all the members (Figure 2). Figure 2B reveals that motifs 2 (residues 10-19) and 6 (residues 9-13) are similar to the MFS signature that is found respectively, between TMs 2 and 3 (GXXXDRXGRR) (Hirai et al., 2003), and TMS 8 and 9 ([RK]XGR [RK]) (Henderson and Maiden, 1990) of the MFS transport proteins. This demonstrates that DehrP has both the MFS and MHS family-specific motifs and therefore is a member of the two groups. Similar motifs have been reported for Burkholderia caribensis MBA4 haloacid transporters (Deh4p) by Tse et al. (2009).

\section{DehrP full sequence alignment}

Comparative analysis of protein sequences by sequence alignment provides useful information during sequence functional analysis by revealing sequence-function relationships (Liò and Bishop, 2008; Shenoy and Jayaram, 2010). Graphical analysis of DehrP primary sequence with six Metabolite: $\mathrm{H}^{+}$Symporter (MHS) family protein sequences from Burkholderia caribensis MBA4 haloacid permeases (Dehp2 and Deh4p (Yu et al., 2007; Su and Tsang, 2013), E. coli Shikimate transporter (ShiA) (Whipp et al., 1998), E. coli proline/betaine transporter; ProP (MacMillan et al., 1999), E. coli Osmoprotectants transporter (YhjE) (Ly et al., 2004), E. coli a-ketoglutarate permease (KgtP) (Seol and Shatkin, 1992), indicates that DehrP sequence is aligned across the full length of each of the transporters (Figure 3). The remaining six MHS also have similar alignment with DehrP (Musa, 2017). Multiple sequence alignment of DehrP with Agrobacterium sp. NHG3 haloacid transporter (DehP $(98 \%$ sequence identity) (Higgins et al., 2005) and B. caribensis MBA4 haloacid transporters (Deh4p; 67\% identity and Dehp2; 60\% identity) (Su and Tsang, 2013; Yu et al., 2007) shows that haloacid transporter sequences are highly conserved (Figure 4) and all have the MFS motifs.

\section{Evolutionary position of DehrP among the MHS transport proteins}

The phylogenetic tree (Figure 5) shows the evolutionary position of Rhizobium sp. RC1 haloacid permease (DehrP) among the Metabolite: $\mathrm{H}^{+}$Symporters (MHS) family of transporters in the Transmembrane Classification Database (TCDB) (Saier et al., 2016), including Burkholderia caribensis MBA4 haloacid transporters (Deh4p and Dehp2) (Su and Tsang, 2013; Yu et al., 2007). The E. coli a-ketoglutarate permease (KgtP) (Seol and Shatkin, 1992) and putative dicarboxylate transporter (PcaT) (Karimian and Ornston, 1981) from Pseudomonas putida are closely related, while the citrate-proton symporters (TcuC and $\mathrm{CitH}$ ) from Salmonella typhimurium(Lewis et al., 2004) and Klebsiella pneumoniae (Van der Rest et al., 1991) are closely related since they both transport a similar substrate. The three haloacid transporters (DehrP, Deh4p and Dehp2) 


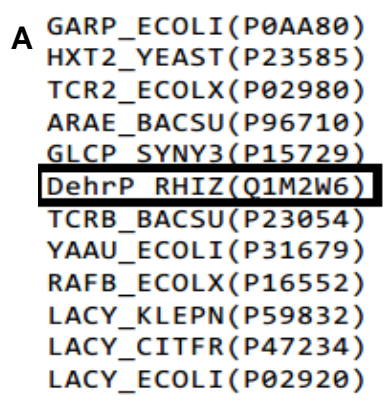

B

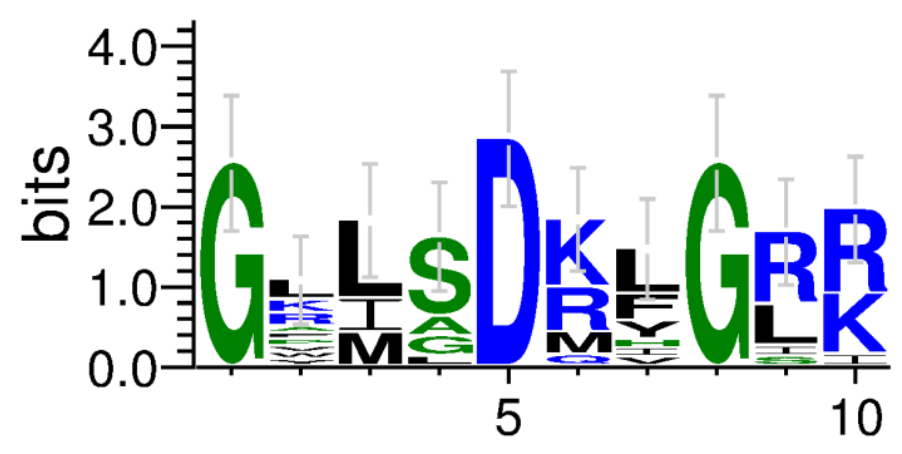

Figure 1: Alignment of Rhizobium sp. RC1 haloacid permease (DehrP) Major Facilitator Superfamily (MFS) motif (GXXXDRXGRR) with other MFS transporters in region connecting transmembrane helices 2 and 3. (A) The conserved MFS signature in DehrP and MFS proteins from E. coli (GARP_ECOLI, TCR2 ECOLX, YJHB_ECOLI, YAAU_ECOLI, RAFB_ECOLX, LACY_ECOLI), C. freundii (LACY_CITFR), K. pneumoniae (LACY_KLEPN), Synechocystis sp. (GLCP_SYNY3), B. subtilis (ARAE_BACSU, and TCRB_BACSU) and S. cerevisiae (HXTT2_YEAST) is highlighted in yellow and the asterisks $\left.{ }^{*}\right)$ indicates conserved residues. The Uniprot accession number of each transporter is shown in bracket. The alignment was done using Clustal omega (Sievers et al., 2011). (B) Logo representation reveals the consensus of the MFS motif among all the MFS proteins as generated by the WebLogo v.3.5 (Crooks et al., 2004).

from Rhizobium sp. RC1 (Jing et al., 2010) and B. caribensis MBA4 (Yu et al., 2007; Su and Tsang, 2013) then joined the dicarboxylate and citrate protonsymporters to form a clade. It appears that KgtP, PcaT, TcuC and $\mathrm{CitH}$, that transport tricarboxylic acid cycle (TCA) intermediates, are relatively closely related to the haloacid transporters. The chloropropionate-induced DehrP (Jing et al., 2010) is more closely related to Dehp2 (Su and Tsang, 2013) than it is to Deh4p (Yu et al., 2007). This is consistent with the findings in a kinetics study by Su and Tsang (2013) that Dehp2 prefers chloropropionate to monochloroacetate while Deh4p has higher affinity for monochloroacetate.

Members of the second clade that are relatively close to the first clade include the $E$. coli osmoregulatory proline/glycine betaine transporter ProP (MacMillan et al., 1999) and the transporter of aromatic compounds, phthalate and 4-methyphthalate, MopB from B. cepacian (Saint and Romas, 1996). These are followed by the $E$. coli transporter aromatic compound shikimate transporter (ShiA) (Whipp et al., 1998), K+ channel (YdfJ) (Tang et al., 2011), putative osmoprotectants transporter (YhjE) (Ly et al., 2004), and the Haemophilus influenza putative hydroxyethylthiazole transporter (ThiU) (Rodionov et al., 2002). Members of the second clade appear to transport diverse substrates and have the ability to transport aromatic compounds. The proton(s) symport activity of CitH (Van der Rest et al., 1991), KgtP (Seol and Shatkin, 1992), and ProP (MacMillan et al., 1999) have been established. On the other hand, the $\mathrm{pH}$-dependent activity of Deh4p and Dehp2 and the inhibition of their MCA transport activity by the un-coupler of transmembrane potential such as protonophore carbonyl cyanide $\mathrm{m}$ chlorophenyl hydrazone (CCCP) have been reported (Su and Tsang, 2013). 
Mal. J. Microbiol. Vol 14(7) 2018, pp. 680-690 DOI: http://dx.doi.org/10.21161/mjm.108417

A

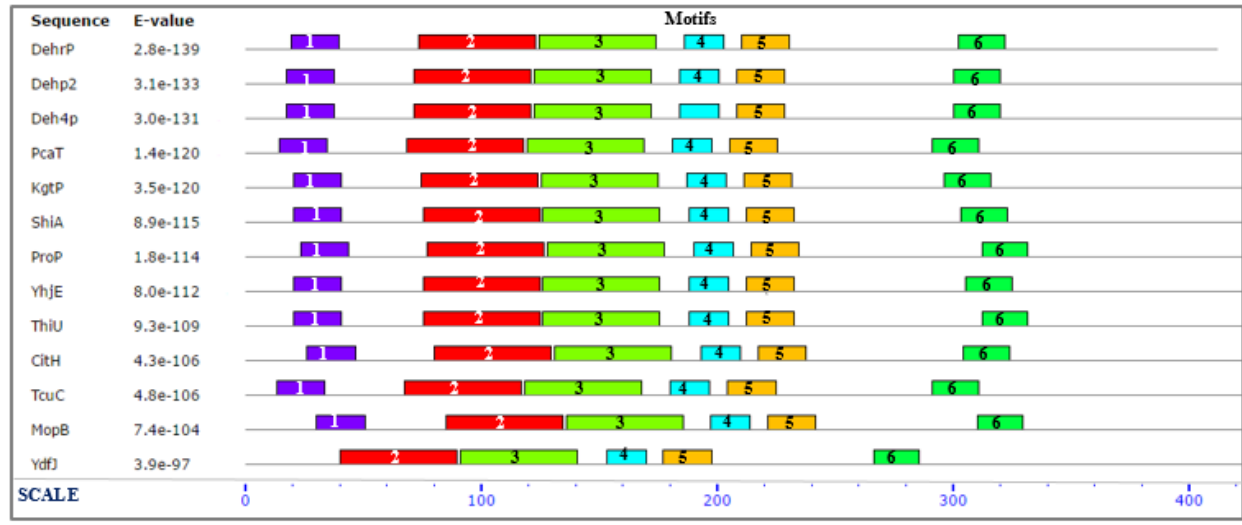

B
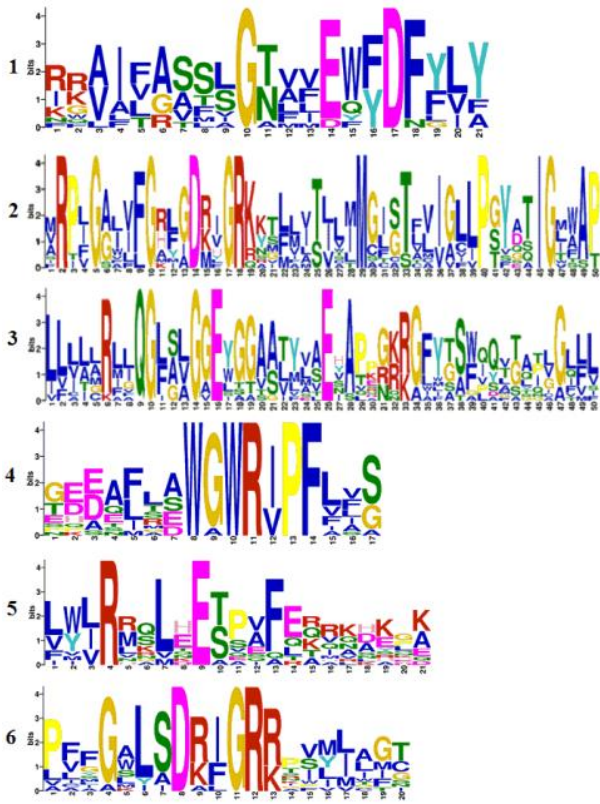

Figure 2: The presence of Metabolite: $\mathrm{H}^{+}$Symporter (MHS) family-specific motifs in DehrP. (A) The relative locations of DehrP six conserved MHS motifs compared with twelve (12) MHS family (\#2.A.1.6) proteins in the Transmembrane Classification Database (TCDB). (B) The signature sequences of the six motifs in logo format. The protein sequences of DehrP (Uniprot ID: Q1M2W6) and the 12 MHS members (TCBD IDs: Dehp2; F8SVK1, Deh4p; Q7X4L6, PcaT; Q52000, KgtP; P0AEX3, ShiA; P76350, ProP; P0C0L7, YhjE; P37643, ThiU; P44699, CitH; P16482, TcuC; P0A2G3, MopB; Q45082, and YdfJ; P77228) in the TCDB were analyzed with the motif-based analysis tools MEME (Bailey and Elkan, 1994) and MAST (Bailey and Gribskov, 1998) in the MEME Suite v.4.11.4. 
Mal. J. Microbiol. Vol 14(7) 2018, pp. 680-690

DOI: http://dx.doi.org/10.21161/mjm.108417
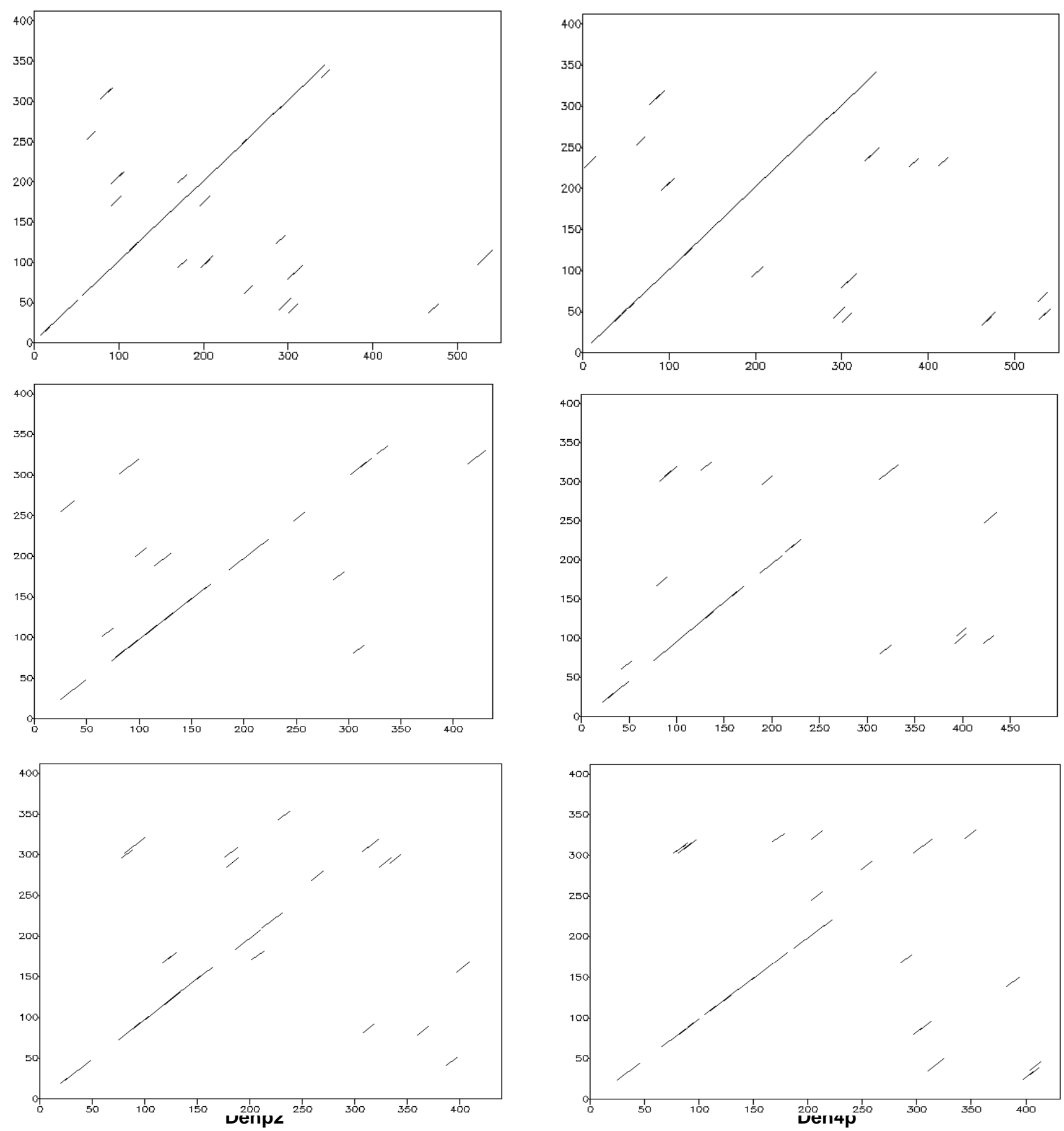

Figure 3: Comparison of DehrP 412 amino acid sequence with the Metabolite: $\mathrm{H}^{+}$Symporter (MHS) family transport protein sequences. DehrP sequence (Uniprot ID: Q1M2W6, x-axis) was compared with six MHS proteins ( $y$-axis) in the Transmembrane Classification Database (TCDB) with the TCDB IDs: Dehp2; F8SVK1, Deh4p; Q7X4L6, ShiA; P76350, ProP; P0C0L7, YhjE; P37643, and KgtP; P0AEX3). The dot plots were generated using Dotmatcher of the EMBOSS (Rice et al., 2000) server with a window size of 10, a threshold of 23 and a default matrix was used. 
Mal. J. Microbiol. Vol 14(7) 2018, pp. 680-690 DOI: http://dx.doi.org/10.21161/mjm.108417
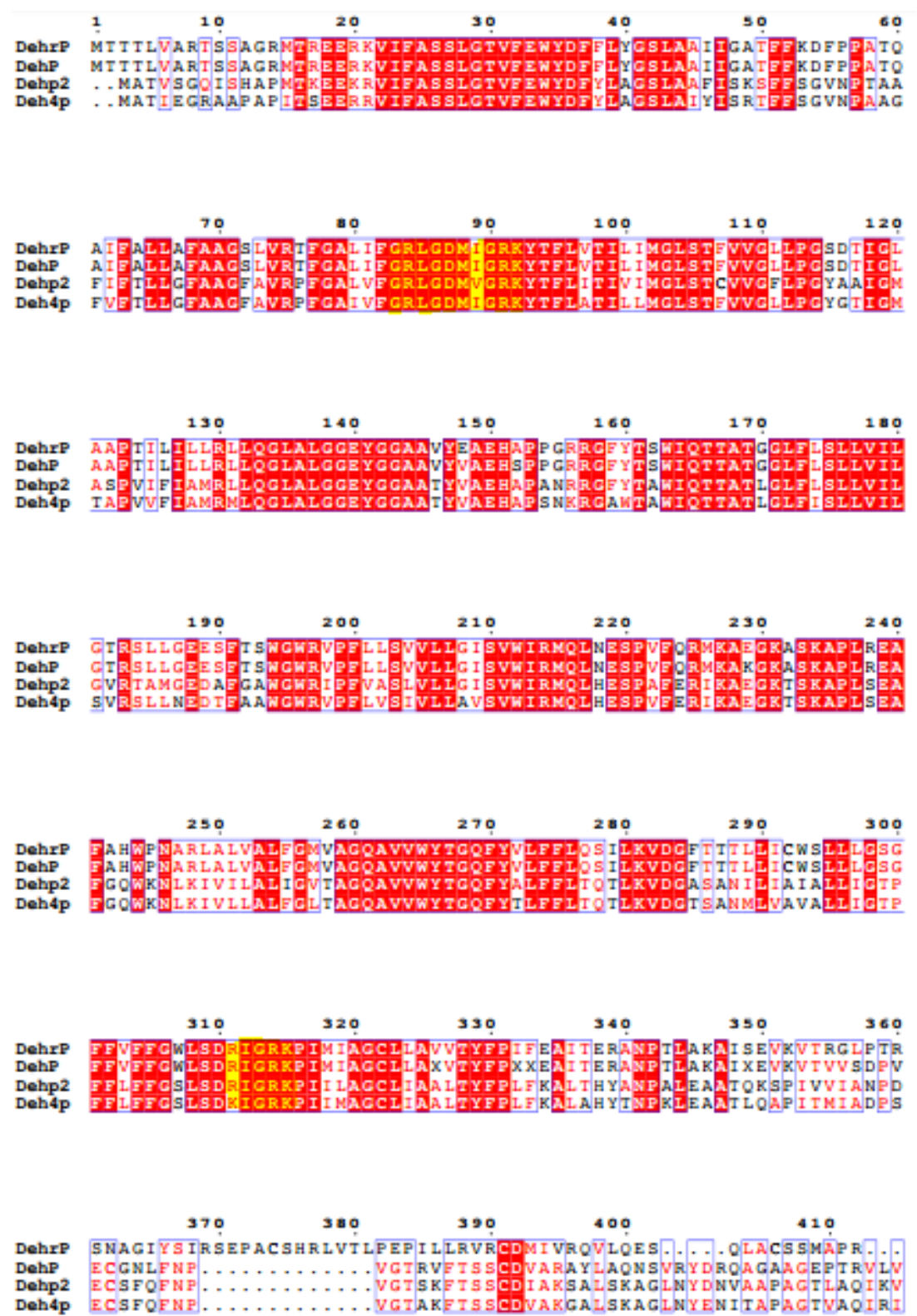

Figure 4: The conserved regions of DehrP and closely related haloacid transporters. DehrP (Uniprot accession number; Q1M2W6 (Jing et al., 2010)) share conserved regions with Agrobacterium sp. NHG3 haloacid transporter (DehP, Uniprot accession number; Q8KLT0 (Higgins et al., 2005) and B. caribensis MBA4 haloacid transporters (Deh4p, Uniprot accession number; A0A0N7JV68 (Yu et al., 2007) and Dehp2, Uniprot accession number; A0A0P0R5D (Su and Tsang, 2013). The highly-conserved regions (red) contains the conserved MFS signature motifs of GXXXDRXGRR and $[R K] X G R[R K]$ shown in yellow highlights. The alignment was done using Clustal Omega (Sievers et al., 2011) and visualized by ESPript v.3.0 (Robert and Gouet, 2014). 


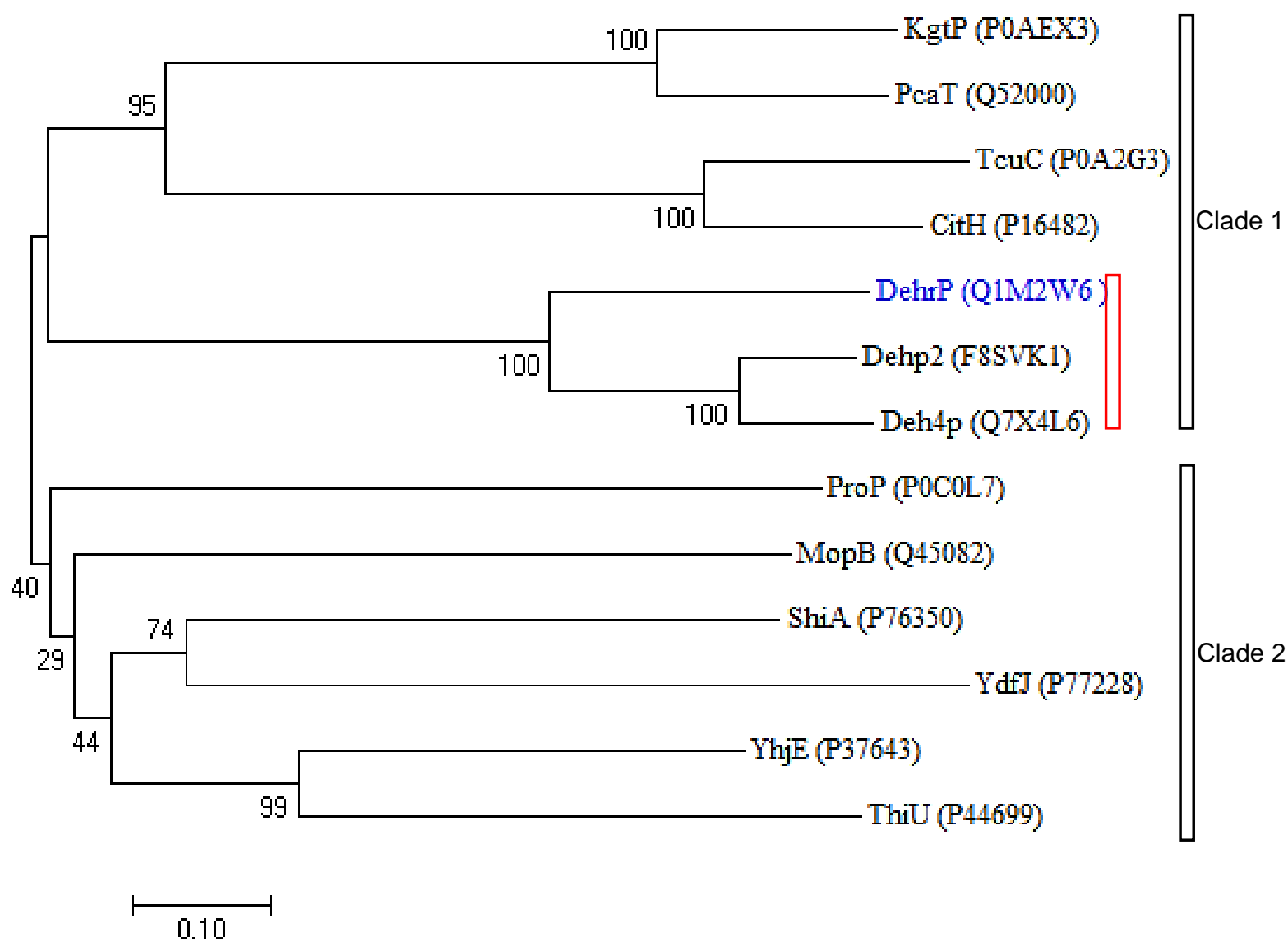

Figure 5: Phylogenetic relationship of DehrP and other Metabolite: $\mathrm{H}^{+}$Symporters (MHS) family members. Haloacid transporters from Rhizobium sp. RC1 (DehrP in blue font) and B. caribensis MBA4 (Dehp2 and Deh4p) are indicated by a red bar. The 12 amino acid sequences were obtained from the transmembrane classification database (TCDB) with the accession numbers shown in brackets. The MHS amino acid sequences were aligned by ClustalW (Larkin et al., 2007), and the tree was constructed by MEGA7 (Kumar et al., 2016) with a neighbor-joining method (Saitou and Nei, 1987). The bootstrap values were calculated with 500 replicates and those above 50 are shown at the nodes. The scale bar represents substitutions per site.

\section{CONCLUSION}

The use of comparative sequence analysis to provide information about sequence-function relationship has become an essential part of bioinformatics. Based on sequence similarity, Rhizobium sp. RC1 haloacid permease (DehrP) is a major facilitator superfamily protein that belongs to the Metabolite: $\mathrm{H}^{+}$Symporter (\#2.A.1.6) subfamily and is closely related to Agrobacterium sp. NHG3 and $B$. caribensis MBA4 haloacid transporters. DehrP has transporter motifs that matches the major facilitator superfamily transport protein motifs. These motifs might function in haloacid transport in Rhizobium sp. RC1. Analysis of the effects of transmembrane electrochemical gradient and $\mathrm{pH}$ on haloacid transport can be used to confirm the symporter activity of DehrP. Threading the sequence of DehrP unto the MFS transport proteins of known crystal structure might provide insight as to whether these motifs are part of the binding site(s) of Rhizobium sp. RC1 haloacid permease.

\section{ACKNOWLEDGMENT}

This work was supported by the Research University Grant (RUG) Vot Q.J130000.2545.12H91, awarded to Universiti Teknologi Malaysia by the Ministry of Education Malaysia. The funding body had no role in the design of the study, analysis, and interpretation of data and in writing of the manuscript. MAM thanks the Kaduna State Government of Nigeria Scholarship for studentship award. 


\section{REFERENCES}

Altschup, S. F., Gish, W., Miller, W., Myers, E. W., and Lipman, D. J. (1990). Basic local alignment search tool. Journal of Molecular Biology 215, (3), 403-410.

Bailey, T. L. and Elkan, C. (1994). Fitting a mixture model by expectation maximization to discover motifs in biopolymers. Proceedings of the International Conference on Intelligent Systems for Molecular Biology 2, 28-36.

Bailey, T. L. and Gribskov, M. (1998). Combining evidence using p-values: Application to sequence homology searches. Bioinformatics 14, (1), 48-54.

Berry, E. K. M., Allison, N., Skinner, A. J., and Cooper, R. A. (1979). Degradation of the selective herbicide 2,2-dichloropropionate (Dalapon) by a soil bacterium. Microbiology 110(1), 39-45.

Berthiaume, C., Gilbert, Y., Fournier-Larente, J., Pluchon, C., Filion, G., Jubinville, E., ... and Charette, S. J. (2014). Identification of dichloroacetic acid degrading Cupriavidus bacteria in a drinking water distribution network model. Journal of Applied Microbiology 116(1), 208-221.

Cairns, S. S. (1994). The cloning and analysis of Rhizobium dehalogenase genes. Doctoral dissertation, University of Leicester, London.

Chaudhry, G. R. and Chapalamadugu, S. (1991). Biodegradation of halogenated organic compounds. Microbiological Reviews 55(1), 59-79.

Consortium, U. (2015). UniProt: A hub for protein information. Nucleic Acids Research 43 (Database issue), D204-212.

Crooks, G. E., Hon, G., Chandonia, J. M., and Brenner, S. E. (2004). WebLogo: A sequence logo generator. Genome Research 14(6), 1188-1190.

Effendi, A. J., Greenaway, S. D. and Dancer, B. N. (2000). Isolation and characterization of 2,3-dichloro-1propanol-degrading Rhizobia. Applied and Environmental Microbiology 66(7), 2882-2887.

Felsenstein, J. (1985). Confidence limits on phylogenies: An approach using the bootstrap. Evolution 39(4), 783791.

Finn, R. D., Coggill, P., Eberhardt, R. Y., Eddy, S. R., Mistry, J., Mitchell, A. L., ... and Salazar, G. A. (2016). The Pfam protein families database: Towards a more sustainable future. Nucleic Acids Research $\mathbf{4 4}$ (Database issue), D279-D285.

Frillingos, S., Sahin-tóth, M., Wu, J. and Kaback, H. R. (1998). Cys-scanning mutagenesis: A novel approach to structure-function relationships in polytopic membrane proteins. The FASEB Journal 12(13), 1281 1299.

Henderson, P. J. and Maiden, M. C. (1990). Homologous sugar transport proteins in Escherichia coli and their relatives in both prokaryotes and eukaryotes. Philosophical Transactions of the Royal Society of London Series B Biological Sciences 326(1236), 391-410.

Higgins, T. P., Hope, S. J., Effendi, A. J., Dawson, S. and Dancer, B. N. (2005). Biochemical and molecular characterisation of the 2,3-dichloro-1-propanol dehalogenase and stereospecific haloalkanoic dehalogenases from a versatile Agrobacterium sp. Biodegradation 16(5), 485-492.

Hirai, T., Heymann, J. A. W., Maloney, P. C. and Subramaniam, S. (2003). Structural Model for 12Helix transporters belonging to the major facilitator superfamily. Journal of Bacteriology 185(5), 17121718.

Huyop, F. Z., Cooper, A. R. A. (2003). A potential use of dehalogenase $D$ (DehD) from Rhizobium $\mathrm{sp}$. for industrial process. Jurnal Teknologi 39(C), 1-8.

Janssen, D. B., Scheper, A., Dijkhuizen, L. and Witholt, B. (1985). Degradation of halogenated aliphatic compounds by Xanthobacter autotrophicus GJ10. Applied and Environmental Microbiology 49(3), 673-677.

Jessen-Marshall, A. E., Paul, N. J. and Brooker, R. J. (1995). The conserved motif, $\mathrm{GXXX}(\mathrm{D} / \mathrm{E})(\mathrm{R} / \mathrm{K}) \mathrm{XG}[\mathrm{X}](\mathrm{R} / \mathrm{K})(\mathrm{R} / \mathrm{K})$, in hydrophilic loop $2 / 3$ of the lactose permease. The Journal of Biological Chemistry 270(27), 16251-16257.

Jing, N., Wahab, R. A., Hamdan, S. and Huyop, F. (2010). Cloning and DNA Sequence Analysis of the Haloalkanoic Permease Uptake Gene from Rhizobium sp. RC 1. Biotechnology 9(3), 319-325.

Karimian, M. and Ornston, L. N. (1981). Participation of the beta-ketoadipate transport system in chemotaxis. Journal of General Microbiology 124(1), 25-28.

Kumar, S., Stecher, G., and Tamura, K. (2016). MEGA7: Molecular evolutionary genetics analysis version 7.0 for bigger datasets. Molecular Biology and Evolution, 33(7), 1870-4.

Larkin, M. A., Blackshields, G., Brown, N. P., Chenna, R., McGettigan, P. A., McWilliam, H., ... and Thompson, J. D. (2007). Clustal W and Clustal X version 2.0. Bioinformatics 23(21), 2947-2948.

Leigh, J., Skinner, A. and Cooper, R. (1986). Isolation and partial characterisation of dehalogenase-deficient mutants of a Rhizobium sp. FEMS Microbiology Letters 36(2-3), 163-166.

Leigh, J. A., Skinner, A. J. and Cooper, R. A. (1988). Partial purification, stereospecificity and stoichiometry of three dehalogenases from a Rhizobium species. FEMS Microbiology Letters 49(3), 353-356.

Lewis, J. A., Horswill, A. R., Schwem, B. E. and Escalante-Semerena, J. C. (2004). The Tricarballylate utilization (tcuRABC) genes of Salmonella enterica serovar Typhimurium LT2. Journal of Bacteriology 186(6), 1629-1637.

Liò, P. and Bishop, M. (2008). Modeling sequence evolution. Bioinformatics: Data, Sequence Analysis and Evolution 255-285.

Ly, A., Henderson, J., Lu, A., Culham, D. E., and Wood, J. M. (2004). Osmoregulatory systems of Escherichia coli: Identification of betaine-carnitinecholine transporter family member BetU and distributions of betU and trkG among pathogenic and nonpathogenic isolates. Journal of Bacteriology 186(2), 296-306. 
MacMillan, S. V., Alexander, D. A., Culham, D. E., Kunte, H. J., Marshall, E. V., Rochon, D., ... and Wood, J. M. (1999). The ion coupling and organic substrate specificities of osmoregulatory transporter ProP in Escherichia coli. Biochimica et Biophysica Acta 1420(1), 30-44.

Marger, M. D. and Saier, M. H. (1993). A major superfamily of transmembrane facilitators that catalyse uniport, symport and antiport. Trends in Biochemical Sciences 18(1), 13-20.

Mitchell, P. (1967). Translocations through natural membranes. Enzymology and Related Areas of Molecular Biology 29, 33-87.

Musa, M. A. (2017). Computational analysis of putative haloalkanoic permease (DehrP) from Rhizobium sp. RC1. Masters dissertation, Universiti Teknologi Malaysia, Malaysia.

Nijenhuis, I., Andert, J., Beck, K., Kastner, M., Diekert, G. and Richnow, H. H. (2005). Stable isotope fractionation of tetrachloroethene during reductive dechlorination by Sulfurospirillum multivorans and Desulfitobacterium sp. strain PCE-S and abiotic reactions with cyanocobalamin. Applied and Environmental Microbiology 71(7), 3413-3419.

Pao, S. S., Paulsen, I. T. and Saier, M. H. (1998). Major facilitator superfamily. Microbiology and Molecular Biology Reviews 62(1), 1-34.

Plewa, M. J., Simmons, J. E., Richardson, S. D. and Wagner, E. D. (2010). Mammalian cell cytotoxicity and genotoxicity of the haloacetic acids, a major class of drinking water disinfection by-products. Environmental and Molecular Mutagenesis 51(8-9), 871-878.

Reddy, V. S., Shlykov, M. A., Castillo, R., Sun, E. I. and Saier, M. H. (2012). The major facilitator superfamily (MFS) revisited. FEBS Journal 279(11), 2022-2035.

Rice, P., Longden, I. and Bleasby, A. (2000). EMBOSS: The European molecular biology open software suite. Trends in Genetics 16(6), 276-277.

Robert, X. and Gouet, P. (2014). Deciphering key features in protein structures with the new ENDscript server. Nucleic Acids Research 42(Web Server issue), W320-324.

Rodionov, D. A., Vitreschak, A. G., Mironov, A. A. and Gelfand, M. S. (2002). Comparative genomics of thiamin biosynthesis in procaryotes new genes and regulatory mechanisms. Journal of Biological Chemistry 277(50), 48949-48959.

Saier, M. H., Reddy, V. S., Tsu, B. V., Ahmed, M. S., Li, C. and Moreno-Hagelsieb, G. (2016). The transporter classification database (TCDB): Recent advances. Nucleic Acids Research 44(Database issue), D372D379.

Saint, C. P. and Romas, P. (1996). 4-Methylphthalate catabolism in Burkholderia (Pseudomonas) cepacia Pc701: A gene encoding a phthalate-specific permease forms part of a novel gene cluster. Microbiology 142(Pt 9), 2407-2418.

Saitou, N. and Nei, M. (1987). The neighbor-joining method: A new method for reconstructing phylogenetic trees. Molecular Biology and Evolution 4(4), 406-425.
Schaffer, A. A., Aravind, L., Madden, T. L., Shavirin, S., Spouge, J. L., Wolf, Y. I., ... and Altschul, S. F. (2001). Improving the accuracy of PSI-BLAST protein database searches with composition-based statistics and other refinements. Nucleic Acids Research 29(14), 2994-3005.

Seol, W. and Shatkin, A. J. (1992). Escherichia coli alpha-ketoglutarate permease is a constitutively expressed proton symporter. Journal of Biological Chemistry 267(9), 6409-6413.

Shenoy, S. R. and Jayaram, B. (2010). Proteins: sequence to structure and function-current status. Current Protein and Peptide Science 11(7), 498-514.

Sievers, F., Wilm, A., Dineen, D., Gibson, T. J., Karplus, K., Li, W., ... and Higgins D. G. (2011). Fast, scalable generation of high-quality protein multiple sequence alignments using Clustal Omega. Molecular Systems Biology 7(1), 539.

Slater, J. H., Weightman, A. J. and Hall, B. G. (1985). Dehalogenase genes of Pseudomonas putida PP3 on chromosomally located transposable elements. Molecular Biology and Evolution 2(6), 557-567.

Stringfellow, J. M., Cairns, S. S., Cornish, A. and Cooper, R. A. (1997). Haloalkanoate dehalogenase II (DehE) of a Rhizobium sp.- molecular analysis of the gene and formation of carbon monoxide from trihaloacetate by the enzyme. European Journal of Biochemistry 250(3), 789-793.

Strotmann, U. J., Pentenga, M., and Janssen, D. B. (1990). Degradation of 2-chloroethanol by wild type and mutants of Pseudomonas putida US2. Archives of Microbiology 154, (3), 294-300.

Su, X. and Tsang, J. S. (2013). Existence of a robust haloacid transport system in a Burkholderia species bacterium. Biochimica et Biophysica Acta 1828(2), 187-192.

Su, X., Deng, L., Kong, K. F. and Tsang, J. S. (2013). Enhanced degradation of haloacid by heterologous expression in related Burkholderia species. Biotechnology and Bioengineering 110(10), 26872696.

Tamura, N., Konishi, S., Iwaki, S., Kimura-Someya, T., Nada, S. and Yamaguchi, A. (2001). Complete cysteine-scanning mutagenesis and site-directed chemical modification of the Tn10-encoded metaltetracycline/ $\mathrm{H}_{+}$antiporter. Journal of Biological Chemistry 276(23), 20330-9

Tang, G., Jiang, B., Huang, Y., Fu, M., Wu, L. and Wang, R. (2011). Identification of a novel bacterial $\mathrm{K}_{+}$ channel. The Journal of Membrane Biology 242(3), 153-164.

Tse, Y. M., Yu, M. and Tsang, J. S. (2009). Topological analysis of a haloacid permease of a Burkholderia sp. bacterium with a PhoA-LacZ reporter. BMC Microbiology 9, 233.

van den Wijngaard, A. J., Wind, R. D. and Janssen, D.B. (1993). Kinetics of bacterial growth on chlorinated aliphatic compounds. Applied and Environmental Microbiology 59(7), 2041-2048. 
Malays. J. Microbiol. Vol 14(7) 2018, pp. 624-631

DOI: http://dx.doi.org/10.21161/mjm.111217

van der Ploeg, J. and Janssen, D. B. (1995). Sequence analysis of the upstream region of $\mathrm{dhlB}$, the gene encoding haloalkanoic acid dehalogenase of Xanthobacter autotrophicus GJ10. Biodegradation 6(3), 257-263.

van der Ploeg, J., Willemsen, M., van Hall, G. and Janssen, D. B. (1995). Adaptation of Xanthobacter autotrophicus GJ10 to bromoacetate due to activation and mobilization of the haloacetate dehalogenase gene by insertion element IS1247. Journal of Bacteriology 177(5), 1348-1356.

Van der Rest, M. E., Abee, T., Molenaar, D. and Konings, W. N. (1991). Mechanism and energetics of a citrate-transport system of Klebsiella pneumoniae. European Journal of Biochemistry 195(1), 71-77.

Whipp, M. J., Camakaris, H. and Pittard, A. J. (1998). Cloning and analysis of the shiA gene, which encodes the shikimate transport system of Escherichia coli K12. Gene 209(1-2), 185-192.

Yu, M., Faan, Y. W., Chung, W. Y. and Tsang, J. S. (2007). Isolation and characterization of a novel haloacid permease from Burkholderia cepacia MBA4. Applied Environmental Microbiology 73(15), 48744880. 